IBIMA Publishing

Journal of Radiology Research and Practice

http://www.ibimapublishing.com/journals/RADI/radi.html

Vol. 2015 (2015), Article ID 312482, 6 pages

DOI: $10.5171 / 2015.312482$

Research Article

\title{
Single Coronary Artery
}

\author{
Jonszta Tomas ${ }^{1}$, Pleva Leos ${ }^{2}$, Krivankova Katerina ${ }^{3}$ and Pelikan Anton ${ }^{4}$ \\ ${ }^{1}$ Institute of Radiology, Ostrava University Hospital, Czech Republic \\ Medical Faculty, University of Ostrava, Czech Republic \\ ${ }^{2}$ Cardiovascular Department, Ostrava University Hospital, Czech Republic \\ Medical Faculty, University of Ostrava, Czech Republic \\ ${ }^{3}$ Institute of Radiology, Ostrava University Hospital, Czech Republic \\ ${ }^{4}$ Department of Surgery, Ostrava University Hospital, Czech Republic
}

Correspondence should be addressed to: Jonszta Tomas; jonszta@post.cz

Received date: 12 January 2014; Accepted date: 9 March 2015; Published date: 1 June 2015

Academic Editor: George D. Giannoglou

Copyright (C) 2015. Jonszta Tomas, Pleva Leos, Krivankova Katerina and Pelikan Anton. Distributed under Creative Commons CC-BY 4.0

\begin{abstract}
Single coronary artery is a rare congenital coronary artery anomaly. It can be associated with myocardial ischemia, increased risk of sudden cardiac death or other congenital cardiac anomalies. The authors present a case of isolated single coronary artery with origin of the right coronary artery (RCA) from the left circumflex artery (LCx) with benign course, found on coronary angiography and computed tomography coronary angiography. A 79-years old female patient presenting with shortness of breath and atypical chest pain was examined. No significant stenosis was detected, and the patient was dismissed from the hospital with adjustment of her medication only. The contribution of conventional coronary angiography, and cardiac computed tomography for meticulous anatomic depiction of coronary arteries is discussed.
\end{abstract}

Keywords: Coronary anomaly, computed tomography coronary angiography, extreme left coronary dominance

\section{Introduction}

Isolated coronary artery anomalies are found in approximately $1 \%$ of patients on coronary arteriograms and $0,3 \%$ of patients at autopsy. ${ }^{1-3}$ In as little as $0,024 \%$ of people, a single coronary artery is formed.4,5 This variation is usually benign, but may be associated with congenital heart disease such as tetralogy of Fallot, transposition of the great arteries, truncus arteriosus, and coronary artery fistula. ${ }^{6}$ We present a patient with single left coronary artery detected by coronary angiography and further depicted by cardiac CT.

\section{Case Description}

A 79-years old female with progressive shortness of breath, occasional palpitations, atypical chest pain and diminishing tolerance of exertion was scheduled for diagnostic coronary angiography. On physical examination, the blood pressure was $130 / 90$ 
mmHg, and her pulse rate was 68. The electrocardiogram displayed atrial fibrillation, slight ST depression and T-wave inversion in V4-6. The transthoracic echocardiographic examination proved normal systolic function of the heart with minor mitral and tricuspid valves regurgitation. She has also been treated for hypertension for a long time, and had pacemaker implanted in 2006 and repositioned in 2009. She suffered from chronic atrial fibrillation and dyslipidemia. She was on anticoagulation therapy with Warfarin. Previous chest X-ray revealed atherosclerotic plaques in the aortic walls.

The course of the cardiac catheterization was uneventful with no signs of significant coronary arteries stenosing process. The right coronary artery origin was not detected during the procedure. Both attempts of selective catheterization and aortography using power-injector were negative for right coronary artery ostium detection. Instead, a large single left coronary artery was discovered with large calibre left circumflex artery continuing distally, crossing the crux cordis and following the right atrioventricular groove, supplying the right coronary artery territory branches. (Figure 1, 2) This retrocardiac course of the artery is considered a benign variant. Therefore, subsequent coronary computed tomography angiography was ordered to depict thoroughly the cardiac anatomy, and exclude any coincidental anomaly of the chest arteries. The examination was performed without prior beta blockers administration on the single source 128- slice CT, with standard cardiac CT scanning mode and retrospective gating protocol. The CT device employed was Siemens Definition AS+, Erlangen, Germany and the datasets were post-processed using Siemens Circulation and Aquarius Terarecon software. The quality of the images was good and allowed complete depiction of this variation. No accompanying great vessels anomalies were detected. (Figures 3-5) The V/Q lung scan was negative for pulmonary embolization. No correlation between angiographic findings and the patient's symptoms was found. The patient was treated conservatively; minor changes in her antihypertensive medication were only done, and the patient's clinical status has been satisfactory so far.

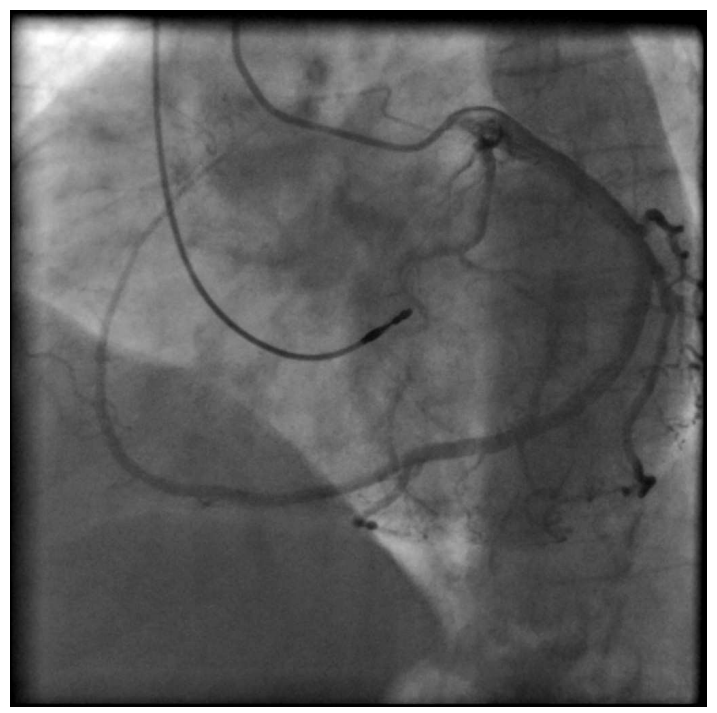

Figure 1: Conventional Coronary Angiography: Large- Calibre, Long Left Circumflex Artery Supplying the Right Ventricular and Atrial Branches, Right Coronary Artery Originating as a Distal Continuation of the LCx. 


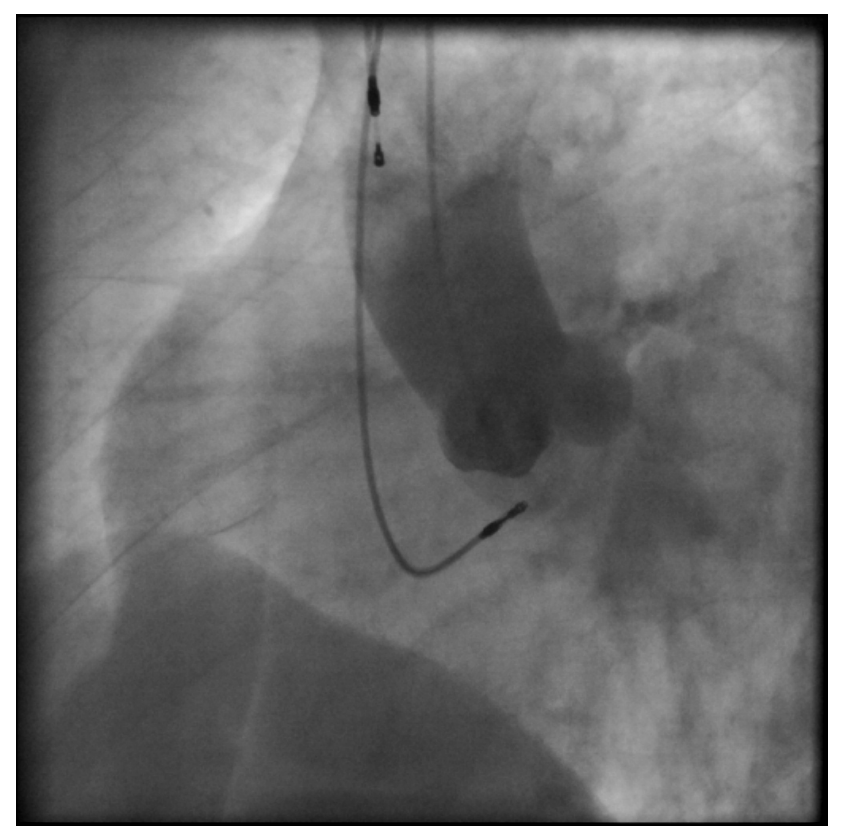

Figure 2: Conventional Aortography: Origin of the Single Left Coronary Artery from the Left Sinus of Valsalva. The Typical Ostium of the RCA from Right Sinus of Valsalva is Missing.

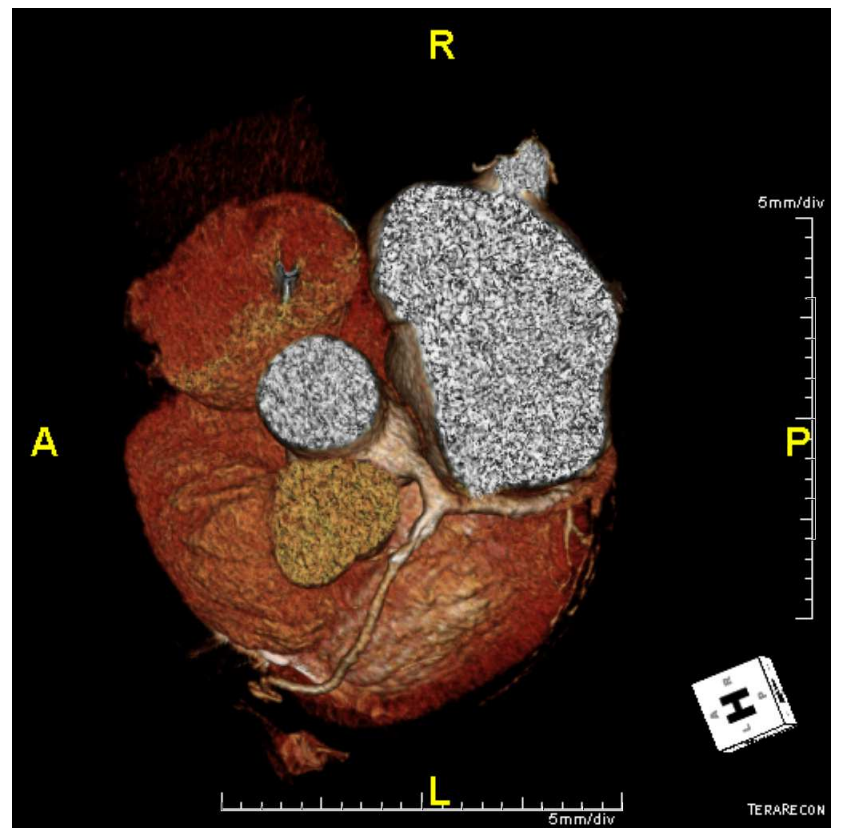

Figure 3: Coronary CT Angiography- VRT Reconstruction: Origin of the Left Coronary Artery from Left Sinus of Valsalva. Right Coronary Artery Doesn't Originate from the Right Sinus of Valsalva. 


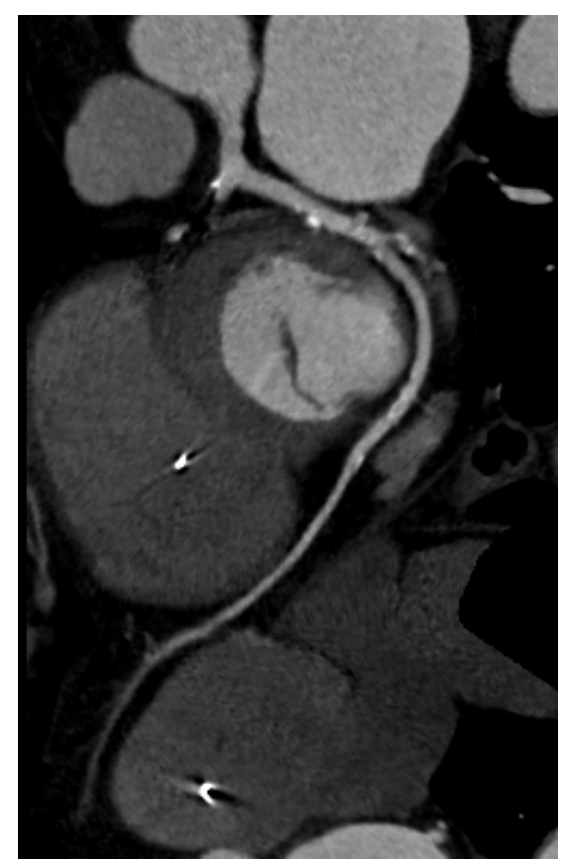

Figure 4: Coronary CT Angiography- MIP Curved Reconstruction along the Long Axis of the LCx-RCA: Atherosclerotic Plaques without Significant Artery Stenosis.

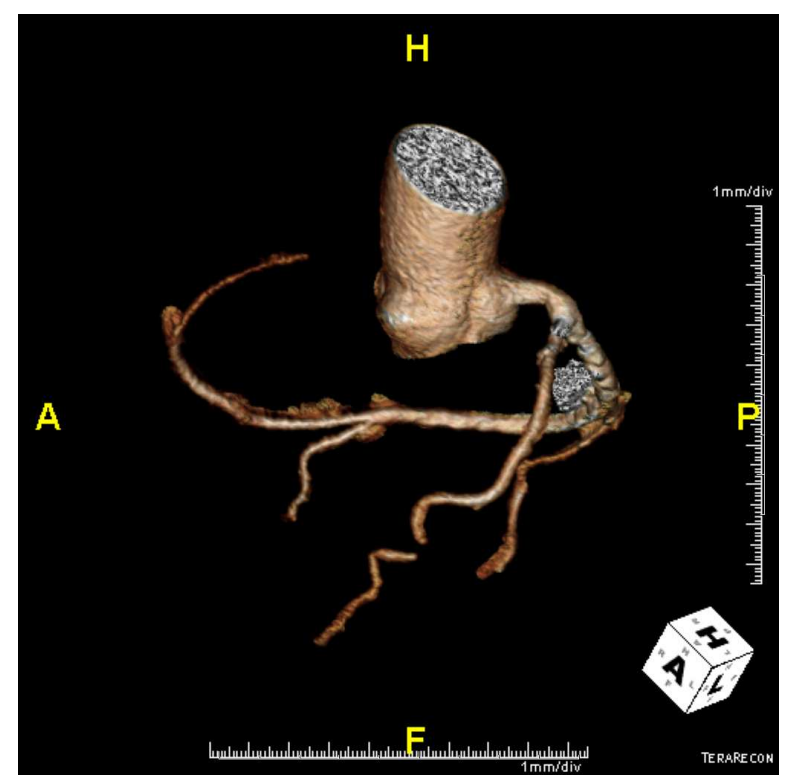

Figure 5: Coronary CT Angiography- Subtracted VRT Reconstruction: Demonstrating the Long Left Circumflex Artery Supplying the Right Ventricular and Atrial Branches.

\section{Discussion}

Coronary artery variations or anomalies are relatively often encountered in cardiac patients. The incidence is about 1 in 100 patients undergoing cardiac catheterization. Coronary arteries variations are classified as anomalies with or without shunt or congenital aneurysms. ${ }^{7,8}$

In our patient, a single left coronary artery was detected, which is considered a very rare finding. According to the scientific literature, a single coronary artery occurs in $0,024 \%$ - 
$0,066 \%$ of people. ${ }^{4,5}$ In the large group of 8235 patients examined by coronary angiography published by Donaldson et Al. (1982), there was the right coronary artery entirely replaced by a large circumflex artery only in 4 patients. ${ }^{9}$ Single coronary artery is defined as only one coronary artery originating from sinus of Valsalva, supplying the entire heart, regardless of its distribution pattern. Depending on the course of the RCA, this finding may be benign or may place the patient at increased cardiac risk. If the anomalous artery traverses within the aortic wall or between the aorta and pulmonary artery, it can be associated with myocardial ischemia and increased risk for sudden cardiac death.

In our patient, the RCA was present as the distal continuation of the LCx behind the atrioventricular (AV) valves, that is called the posterior (to the AV valves) course of an ectopically originating RCA from the left coronary artery. This variant is considered to be benign and classified as Lipton (1979) L1 anomaly. According to a newer classification by Yamanaka et Al. (1990), the L1 group of single coronary artery anomalies usually has a benign clinical course. ${ }^{1}$ This configuration can also be called extreme left dominance. Normally, approximately $85 \%$ of the general population is right-dominant, $8 \%$ are leftdominant and $7 \%$ are co-dominant. ${ }^{10}$

Traditionally, most radiographic descriptions of the coronary arteries have been based on the cine coronary angiograms performed by coronary ostia catheterization, and direct contrast media injection. In the last decade, the cardiac CT as a complementary imaging modality has brought new dimensions in coronary vessels imaging. It provides detailed anatomic information on coronary origin, course and branching and allows evaluation of all great mediastinal vessels. Various postprocessing methods enable also vessel walls evaluation. ${ }^{11,12}$ The combination of the two above mentioned methods brings most comprehensive evaluation of the coronary anatomy.

However, rarely the single coronary artery with origination of the RCA from the distal LCX is, there can be found a handful of case reports in the recent scientific literature, that demonstrate the same or similar findings, that we encountered in our patient, as well as very useful comments on classification and nomenclature. ${ }^{13-17}$

\section{Conclusion}

Coronary artery anomalies should be regarded as a diverse group of congenital disorders, whose manifestations and pathophysiological mechanisms are highly variable. Some of the anomalies like myocardial bridges are surely encountered in more than $1 \%$ of patients, and probably should be considered normal variant. Some other may have life-threatening consequences or may be encountered with other cardiac anomalies. The cardiac CT plays a key role in diagnostic algorithm of coronary artery variations imaging, and allows perfect anatomic depiction of the coronary artery course.

\section{References}

1. Yamanaka, O. \& Hobbs, R. E. (1990). "Coronary Artery Anomalies in 126,595 Patients Undergoing Coronary Arteriography," Catheterization and Cardiovascular Diagnosis, 21 28-40.

2. Alexander, R. W. \& Griffith, G. C. (1956). "Anomalies of the Coronary Arteries and Their Significance," Circulation, 14 800-805.

3. Angelini, P., Velasco, J. A. \& Flamm, S. (2002). "Coronary Anomalies: Incidence, Pathophysiology, and Clinical Relevance," Circulation, 105 2449-2454.

4. Lipton, M. J., Barry, W. H., Obrez, I. et Al. (1979). "Isolated Single Coronary Artery: Diagnosis, Angiographic Classification, and Clinical Significance," Radiology, 130 39-47

5. Desmet, W., Vanhaecke, J., Vrolix, M. et al. (1992). "Isolated Single Coronary Artery: A Review of 50,000 Consecutive Coronary Angiographies," European Heart Journal, 13 1637-1640.

6. Memisoglu, E., Hobikoglu, G., Tepe, M. S. et al. (2005). "Congenital Coronary Anomalies in 
Adults: Comparison of Anatomic Course Visualized by Catheter Angiography and Electron Beam CT," Catheterization and Cardiovascular Interventions, 66 34-42.

7. Fiss, D. M. (2007). "Normal Coronary Anatomy and Anatomic Variations," Applied Radiology, vol. 36 (1) 14-26.

8. Morettin, L. B. (1976). "Coronary Arteriography: Uncommon Observations," Radiologic Clinics of North America, 14 189208.

9. Donaldson, R. M. \& Raphael, M. J. (1982). "Missing Coronary Artery- Review of Technical Problems in Coronary Arography Resulting from Anatomical Variants," British Heart Journal, 47 62-70.

10. Gensini, G. G. (1975). 'Coronary Arteriography,' Mount Kisco, NY: Futura Publishing Co, 260-274.

11. O'Brien, J. P., Srichai, M. B., Hecht, E. M. et Al. (2007). "Anatomy of the Heart at Multidetector CT: What the Radiologist Needs to Know," RadioGraphics, 27 1569-1582.

12. Rajiah, P., Halliburton, S. S., Scott, F. D. et Al. (2012). "Strategies for Dose Reduction in
Cardiovascular Computed Tomography," Applied Radiology, 41 (7-8) 10-15.

13. Desai, A. V., Parikh, P. \& La Barbera, M. (2013). "Anomalous Right Coronary Artery Originating from Left Circumflex Artery: An Unusual Single Coronary Artery Anomaly," Cath Lab Digest 21 (2) 22.

14. Chung, S. K., Lee, S. J., Park, S. H. et Al. (2010). "An Extremely Rare Variety of Anomalous Coronary Artery: Right Coronary Artery Originating from the Distal Left Circumflex Artery," Korean Circulation Journal 2010; 40(9):465-7.

15. Andreou, A. Y., Tryfonos, A., Christodoulou, C. et Al. (2012). "Isolated single Coronary Artery with Dual Right Coronary Artery Distribution. A Rare Anatomical Variation," Herz. 2012; 37(4):432-5.

16. Angelini, P. (2007). "Coronary Artery Anomalies: An Entity in Search of an Identity," Circulation. 2007 Mar 13; 115(10):1296-305.

17. Angelini, P. (2008). “An Illustrative Case of a Frequently-Held Misconception: The "Absent" RCA," Journal of Invasive Cardiology 2008; $20(7): 384$. 